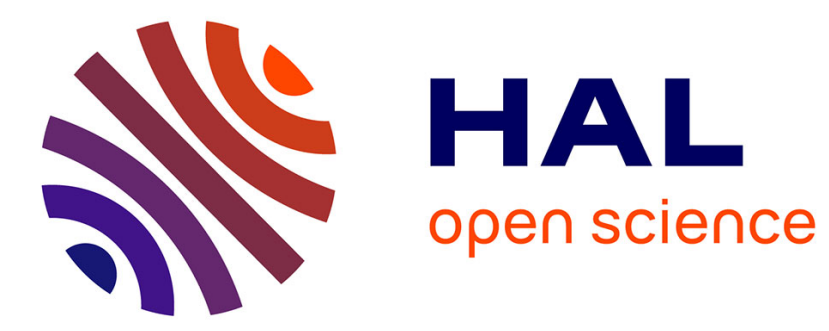

\title{
Light transmission by subwavelength annular aperture arrays in metallic films
}

\author{
F.I. Baida, D. van Labeke
}

\section{To cite this version:}

F.I. Baida, D. van Labeke. Light transmission by subwavelength annular aperture arrays in metallic films. Optics Communications, 2002, 209 (1-3), pp.17-22. 10.1016/S0030-4018(02)01690-5 . hal00097824

\section{HAL Id: hal-00097824 \\ https://hal.science/hal-00097824}

Submitted on 28 Apr 2021

HAL is a multi-disciplinary open access archive for the deposit and dissemination of scientific research documents, whether they are published or not. The documents may come from teaching and research institutions in France or abroad, or from public or private research centers.
L'archive ouverte pluridisciplinaire HAL, est destinée au dépôt et à la diffusion de documents scientifiques de niveau recherche, publiés ou non, émanant des établissements d'enseignement et de recherche français ou étrangers, des laboratoires publics ou privés. 


\title{
Light transmission by subwavelength annular aperture arrays in metallic films
}

\author{
F.I. Baida*, D. Van Labeke \\ Laboratoire d'Optique P.M. Duffieux, Centre National de La Recherche Scientifique Unité Mixte de Recherche 6603, Institut de \\ Microtechniques de Franche-Comté, Université de Franche-Comté, 25030 Besançon Cedex, France
}

\begin{abstract}
We study the spectral response of a metallic film with an engraved 2D periodic structure of annular apertures. We show that an enhanced transmission can be obtained and can reach $90 \%$. The variation of the spectral response as a function of the film thickness from $150 \mathrm{~nm}$ to a value of $1.8 \mu \mathrm{m}$ is also studied. We show that a guided mode in the subwavelength coaxial structure is responsible for this large transmission. A wavelength cut-off exists. We study the transmission characteristics on the optical and geometrical parameters of the array (period, coaxial radii, incident polarization and dielectric constants) using a 3D-FDTD algorithm.
\end{abstract}

Keywords: Cavity; Diffraction; Grating; Subwavelength resolution; Surface plasmon

\section{Introduction}

Since the publication of Ebbesen et al. [1], many experimental and theoretical studies were carried out in order to determine the physical origin of the extraordinary enhanced transmission reported in that paper. A circular aperture array in a metallic film (gold) can lead to a large transmission comparing to the hollow-surface to total-surface ratio (factor 5). Surface plasmon resonances [2-5] or cavity hole resonances [6-9] have been proposed to

\footnotetext{
${ }^{*}$ Corresponding author. Tel: +33-3-81-66-63-02; fax: $+33-3-$ 81-66-64-23.

E-mail address: fbaida@univ-fcomte.fr (F.I. Baida).
}

be at the origin of this phenomenon. This transmission enhancement is similar to what is observed with frequency selective surface (FSS) components in the spectral region from near-infrared to microwave wavelengths $[10,11$ and references therein]. Those components are intended for a broad domain of applications such as Fabry-Pérot interferometers, filters, couplers for laser cavity output or simply as polarizers. Their spectral responses are directly linked to their geometrical parameters i.e. their thickness, size, period and especially their shape. This last point is at the origin of this study. We propose a new structure showing an interesting spectral response in the visible range of the spectrum. Indeed, to increase the transmission of a metal film perforated with 
subwavelength apertures, it is intuitive to consider a structure that has no cut-off frequency in the desired spectral field. This property is verified for the transverse electromagnetic (TEM) mode in a coaxial waveguide made in perfect metal [12]. Thus, we numerically study, the transmission of light by a structure made with a grating of annular apertures instead of a hole grating. Preliminary results show that such a geometry allows a large increase of the transmission coefficient.

\section{Numerical model and simulations}

The principle of the numerical FDTD method can be found in many textbooks $[13,14]$. Both the spatial area and the time interval are discretized. Our numerical code includes the second order Mur technique to solve the parasitical reflections at the edges of the grid [15]. The metal dispersion is taken into account by using a Drude model to describe the frequency dependence of the permittivity as in reference [16].

The object is composed of a periodic array of annular apertures (see Fig. 1): the external and internal radii are $r_{1}$ and $r_{2}$, respectively. The thickness of the film is $e$ and the periods are $p_{x}$ in

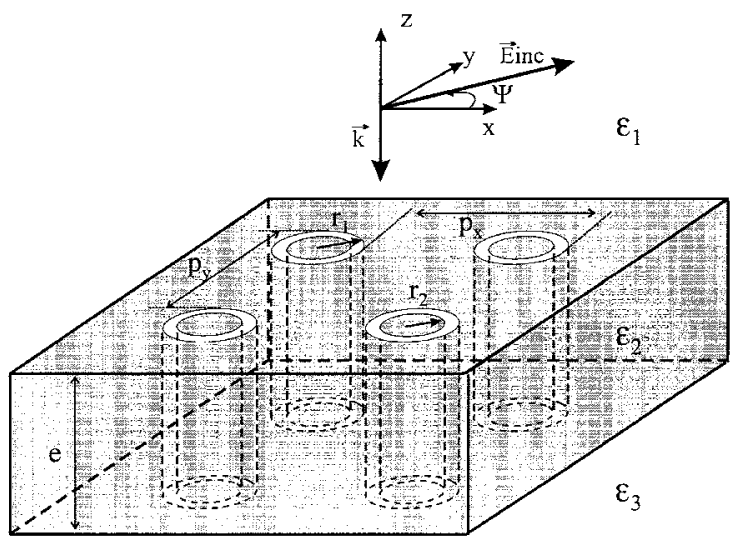

Fig. 1. Schematic of the structure. Annular apertures are made in a metallic film. $r_{1}$ is the external radius, $r_{2}$ is the internal one. $p_{x}$ and $p_{y}$ are the periods in $x$ and $y$ directions, respectively. $\varepsilon_{1}, \varepsilon_{2}$ and $\varepsilon_{3}$ are the incident, metallic and transmission media permittivity. $e$ is the thickness of the metallic film. The normally incident plane wave illuminates the structure. the $x$-direction and $p_{y}$ in the $y$-direction. The permittivity of the incident medium is $\varepsilon_{1}, \varepsilon_{3}$ for the transmission medium and $\varepsilon_{2}$ for metal. In this study, both incident and transmission media are vacuum i.e. $\varepsilon_{1}=\varepsilon_{3}=1$. The dielectric constant of the in-apertures medium is $\varepsilon_{0}$. Its value is set to 1 unless stated otherwise. The illumination is a pulse plane wave which hits the metallic film at normal incidence. The pulse is centered at $600 \mathrm{~nm}$ and has a temporal width that corresponds to a wide spectrum covering all the visible range. The polarization is linear and it is described by the angle $\Psi$ from the $x$-axis (see Fig. 1).

Fig. 2 shows the spectral responses (efficiency of the transmitted zero-order) of a silver structure when $r_{1}=75 \mathrm{~nm}, r_{2}=50 \mathrm{~nm}$ for different thicknesses of the metallic film and for $p_{x}=p_{y}=$ $300 \mathrm{~nm}$.

The spectrum in the visible region is principally composed of two peaks. Their positions and intensities for six metal thicknesses are reported in Table 1. The position of the red peak $\left(\lambda_{2}\right)$ is practically independent of metal thickness. But its intensity decreases when $e$ increases. The other peak $\left(\lambda_{1}\right)$ has more interesting behavior: when the
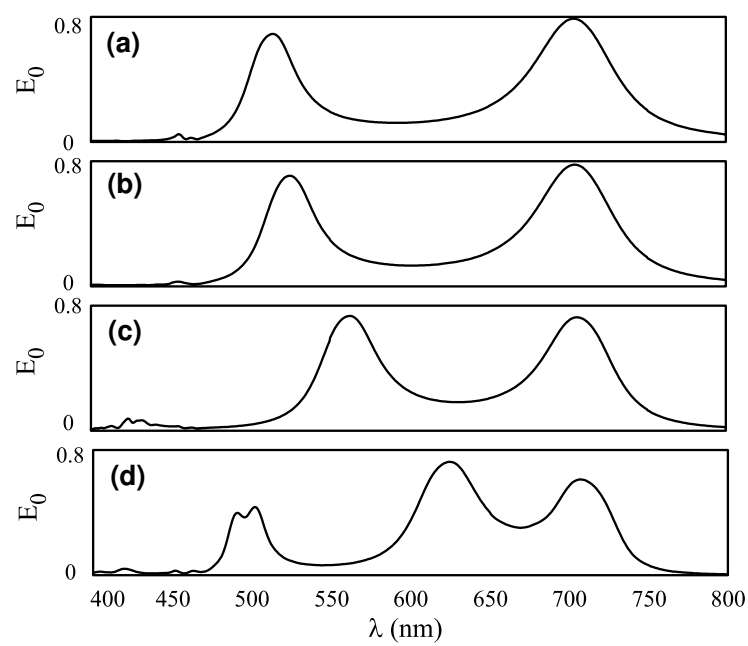

Fig. 2. Zero-order transmission of a structure made in silver film. The parameters of the object are (see Fig. 1): $r_{1}=70 \mathrm{~nm}$, $r_{2}=50 \mathrm{~nm}, p_{x}=p_{y}=300 \mathrm{~nm}, \varepsilon_{1}=\varepsilon_{3}=1$ and the thickness is $e_{1}=150 \mathrm{~nm}$ in (a), $e_{2}=160 \mathrm{~nm}$ in (b), $\varepsilon_{3}=200 \mathrm{~nm}$ in (c) and $\varepsilon_{4}=300 \mathrm{~nm}$ in (d). The illumination is made by a $x$-polarized plane wave at normal incidence. 
Table 1

Positions and transmission values of the two peaks appearing in the spectral response of a silver grating for six metal film thicknesses

\begin{tabular}{lllll}
\hline$e(\mathrm{~nm})$ & $\lambda_{1}(\mathrm{~nm})$ & $E_{0}\left(\lambda_{1}\right)$ & $\lambda_{2}(\mathrm{~nm})$ & $E_{0}\left(\lambda_{2}\right)$ \\
\hline 150 & 514 & 0.6904 & 704 & 0.7883 \\
160 & 525 & 0.7077 & 705 & 0.7783 \\
170 & 535 & 0.7178 & 705 & 0.7656 \\
200 & 563 & 0.7343 & 706 & 0.7253 \\
220 & 579 & 0.7378 & 706 & 0.7002 \\
300 & 625 & 0.7250 & 707 & 0.6127 \\
\hline
\end{tabular}

The optical and geometrical parameters are given in Fig. 2.

metal thickness is increased, it is shifted toward the red region but its intensity remains almost constant. A third peak (a doublet $\lambda=491 \mathrm{~nm}$ and $\lambda=502 \mathrm{~nm}$ ) appears when $e=300 \mathrm{~nm}$.

It is important to notice that the red peak is the largest wavelength that can be transmitted by the structure: the transmission efficiency, at normal incidence, vanishes from $\lambda=1 \mu \mathrm{m}$ to $\lambda=2.8 \mu \mathrm{m}$.

The proposed structure clearly shows a very high transmission in the visible region which may be interesting for several applications as filters, polarizers or resonators especially in nano-optics. Such a structure with nanometric coaxial apertures can be routinely fabricated with available techniques like lift-off process [17] or focused ion beam $[5,18]$. In the following, we modify some geometrical and optical parameters in order to show their influence on the transmission spectrum and to try to interpret it.

\section{Discussion}

The enhanced transmission of aperture gratings are often interpreted in terms of surface plasmon resonance (see [5 and references therein]).

A diffracted wave, resulting from the interaction of the incident light with the structure, has the same wave-vector tangential component as that of the surface plasmon. This leads to the relation $[3,5]$

$\lambda_{1,3}(i, j)=\frac{p_{x} p_{y}}{\sqrt{\left.i^{2} p_{y}^{2}+j^{2} p_{x}^{2}\right)}} \sqrt{\frac{\varepsilon_{1,3} \varepsilon_{2}}{\varepsilon_{1,3}+\varepsilon_{2}}}$,

where $i, j$ are the integers which define the diffracted order. In our case $\left(p_{x}=p_{y}=300 \mathrm{~nm}\right)$, $\varepsilon_{1}=\varepsilon_{3}=1$ and with silver metal, the previous equation has no solution for $\lambda>400 \mathrm{~nm}$. As a consequence, the enhanced transmission of the coaxial grating cannot be interpreted in terms of excitation of horizontal surface plasmons.

The results presented in Fig. 2 and Table 1 suggest that the first peak $\left(\lambda_{1}\right)$ could correspond to a cavity resonance as its position is modified with respect to the metal thickness. When the metal thickness is increased from 150 to $300 \mathrm{~nm}$, the first peak is almost proportionally shifted from 514 to $625 \mathrm{~nm}$.

In order to test this assumption, we have studied the spectral response of a $1.8 \mu \mathrm{m}$ thickness coaxial array. The result is presented in Fig. 3.

The spectrum exhibits 14 peaks which, at a first look, seems compatible with resonance of a longer cavity. However, Fig. 3 spectrum is not that simple to be explained. As in the case of a smaller thickness, there is no transmission above $\lambda \simeq$ $700 \mathrm{~nm}$.

For a single mode cavity resonance, the enhanced transmission is generally described by an Airy-like equation [5,7]:

$t=\frac{4 \tau_{1} \tau_{2} \mathrm{e}^{\mathrm{j} n e w / c}}{1-\rho_{1} \rho_{2} \mathrm{e}^{\mathrm{j} n e w / c}}$,

where $n$ is the effective complex index of the guided mode in the cavities: $\tau_{1}, \tau_{2}, \rho_{1}$ and $\rho_{2}$ are the transmission and reflection coefficients of the two cavities' edges, respectively. Lalanne et al. [7] give

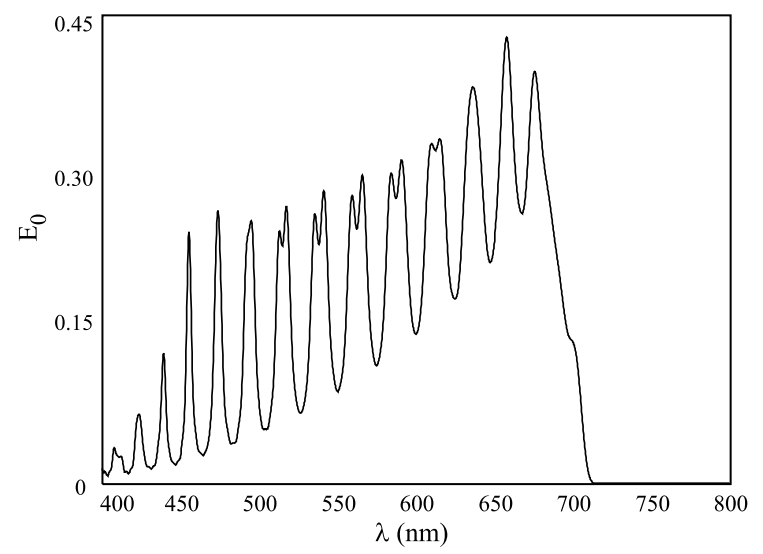

Fig. 3. Spectral response for a $1.8 \mu \mathrm{m}$ thick silver film. All other parameters are the same as in Fig. 2(a). 
the explicit expressions of the parameters of Eq. (1) in the case of a lamellar 1D metallic grating.

We have not been able to fit the spectrum in Fig. 3 using Eq. (1) with constant parameters. The main reasons of the discrepancy are related to the fact that the free spectral range (FSR) is not constant and it is incompatible with the wavelength cut-off $(\lambda \simeq 700 \mathrm{~nm})$.

In a conventional resonant cavity, without damping, the spacing between two successive peaks is related to the $F S R=1 /(2 \operatorname{Real}(n) e)$, where $\operatorname{Real}(n)$ is the real part of the effective complex index $(n)$ of the guided mode. From Fig. 3, an averaged value of the FSR is obtained from the ten first peaks position leading to $\operatorname{Real}(n) \simeq 3.16$. For such a value of the effective index, the cut-off of a cavity with $e=1.8 \mu \mathrm{m}$ will be around $2 e \operatorname{Real}(n)=11.4 \mu \mathrm{m}$. Moreover, the doublets appearing in Figs. 2(d) and 3 cannot be explained by a single mode Airy-like equation. There is also a shoulder in last red peak of the spectrum in Fig. 3, this shoulder, which is at $\lambda \simeq 700 \mathrm{~nm}$ could correspond to the $\lambda_{2}$ peak of spectra in Fig. 2. This $\lambda_{2}$ peak, independent of metal thickness, cannot be interpreted in term of cavity resonance.

The permittivity of metal plays also a role in the spectral response of the structure. Indeed, if silver is replaced by aluminum, the wavelength cut-off is modified. This effect is similar to what observed in a resonant cavity when the phase of the reflection coefficient is modified according to the permittivity of the metallic mirrors. Fig. 4 shows the zero-order transmission of an aluminum structure where the aperture has the same geometrical parameters as in Fig. 2(c). We note that, for aluminum, the two peak structure is maintained but it is shifted toward the blue region. The $\lambda_{2}$ peak is shifted by 130 $\mathrm{nm}$ in comparison with silver.

In a coaxial guide made of a perfect metal, the propagation constants of a TEM mode are independent of the guide radii. This is not the case for TE nor TM modes [12]. Then, it is interesting to point out the influence of the geometrical shape, i.e. the radius values, on the spectral response. Fig. 5 shows the zero-order transmission for a structure, where $r_{1}$ was increased by $10 \mathrm{~nm}$ $\left(r_{1}=85 \mathrm{~nm}\right)$. The thickness is set to $e=200 \mathrm{~nm}$ and all the other parameters are the same as in Fig.

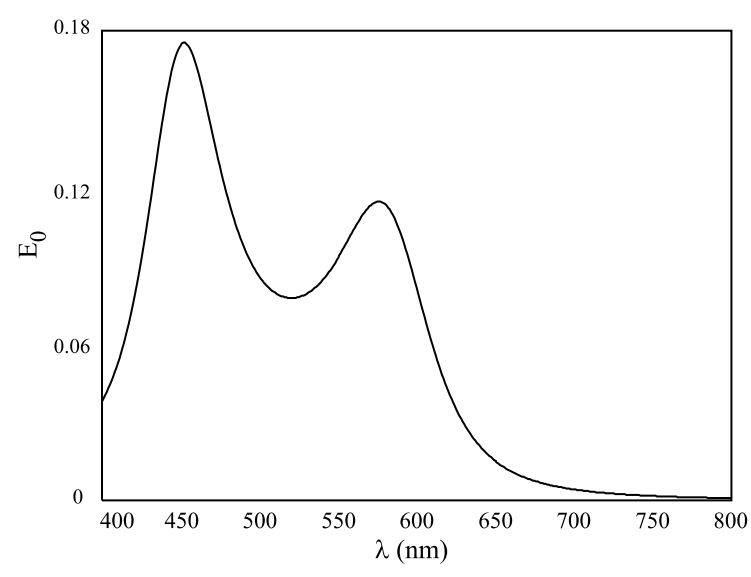

Fig. 4. Spectral response for a structure made in $200 \mathrm{~nm}$ aluminum thick film. $r_{1}=75 \mathrm{~nm}, r_{2}=50 \mathrm{~nm}, p_{x}=p_{y}=300 \mathrm{~nm}$, $\varepsilon_{1}=\varepsilon_{3}=1$. (to be compared with Fig. 2(c)).

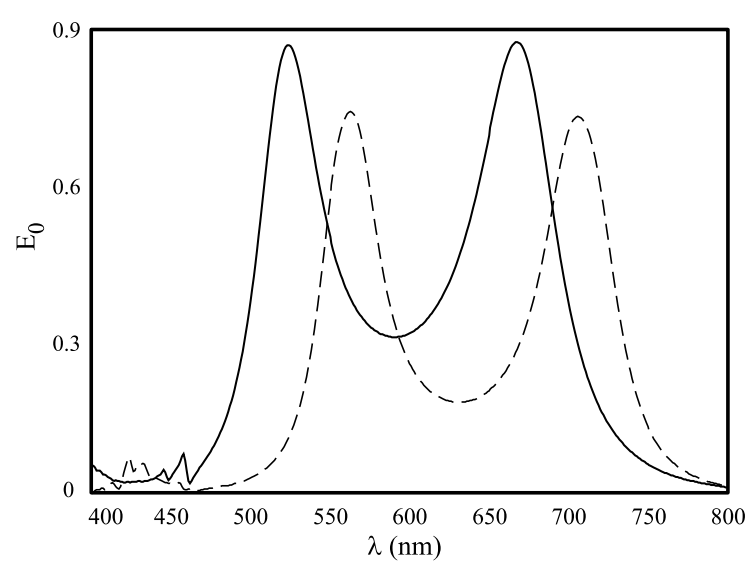

Fig. 5. Spectral responses for two different radii of the annular aperture. The parameters of the object are similar to those of Fig. 2(c) (see Fig. 1) execpted $r_{1}=85 \mathrm{~nm}$ in solid line and $r_{1}=75 \mathrm{~nm}$ in dashed line.

2. We note that the peak structure is shifted toward the blue region of the spectrum by $\Delta \lambda \simeq 39 \mathrm{~nm}$.

Note that, in this case, the maximum of the transmission is higher than with $r_{1}=75 \mathrm{~nm}$. It reaches $90 \%$. This increase in transmission can be hastily connected to the fact that the hollow-surface on total-surface ratio increases. Indeed, this assumption is only valid if the size of the hole is larger than the incident wavelength. 
It is also interesting to see what occurs when the structure period is modified. Fig. 6 shows the spectral responses of the zero-order transmission for four annular aperture arrays where, only the period was modified. The dashed line is the same curve as in Fig. 2(a). When increasing both $x$ and $y$ periods (solid line with squares in Fig. 6), the two peaks are shifted toward the red region. The same behavior is obtained if we only increase the $x$-pe$\operatorname{riod}\left(p_{x} \rightarrow p_{x}+50 \mathrm{~nm}\right.$ for the dotted line of Fig. 6). If we only increase the $y$-period ( $p_{y} \rightarrow p_{y}+50 \mathrm{~nm}$ ), the peaks shift along the blue region (solid line in Fig. 6).

Note that, in all the cases where the periods increase, the peak intensities decrease. The two peak structure is also shifted but it is much more smaller than in the cases of change of radius or metal permittivity.

All the results presented up to now correspond to an illumination where the polarization is directed according to $x$-direction (see Fig. 1). Therefore, it is interesting to study the effect of the polarization direction. In Fig. 7, we plot the spectral responses of the same biperiodic structure studied in Fig. 2(a) where the polarization of the incident beam was tilted by an angle $\Psi$ with the $x$ axis (see Fig. 1).

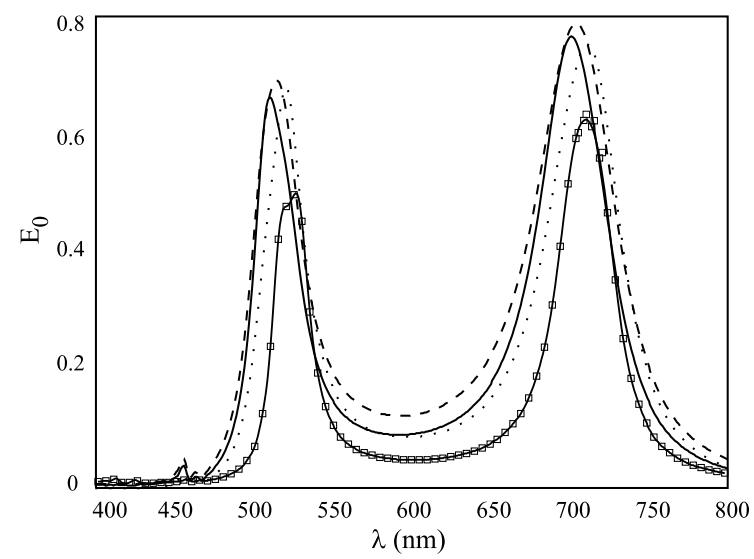

Fig. 6. Zero-order transmission of a structure made in $150 \mathrm{~nm}$ thick silver film. $r_{1}=75 \mathrm{~nm}, \quad r_{2}=50 \mathrm{~nm}, \quad \varepsilon_{1}=\varepsilon_{3}=1$. $p_{x}=p_{y}=400 \mathrm{~nm}$ in solid line with squares, $p_{y}=$ $p_{x}+50=350 \mathrm{~nm}$ in solid line, $p_{x}=p_{y}+50=350 \mathrm{~nm}$ in dotted line and for comparison, $p_{x}=p_{y}=300 \mathrm{~nm}$ in dashed line.

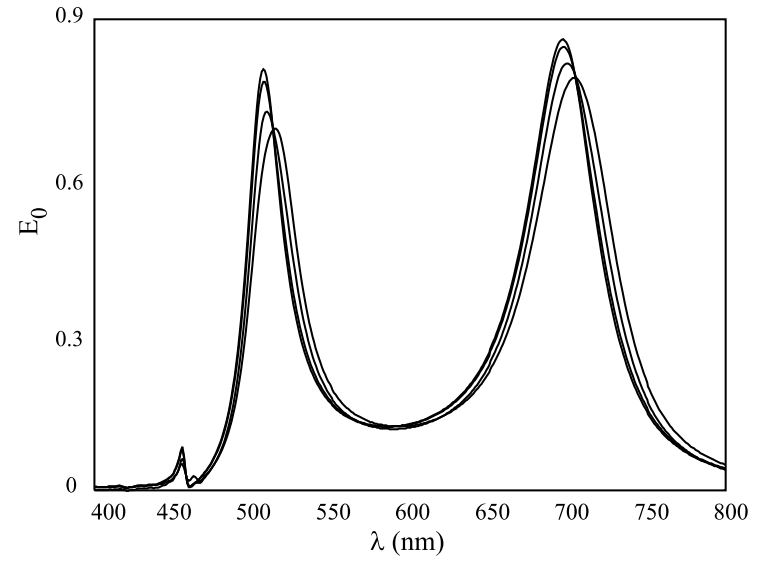

Fig. 7. Zero-order transmission of the same structure studied in Fig. 2(a) when the polarization of the incident beam varies from 0 to $45^{\circ}$ by $15^{\circ}$ steps. The transmission increases with $\Psi$. Note that, because of the symmetry, the same curve is obtained for $\Psi=60^{\circ}$ and $\Psi=30^{\circ}$.

As in the case of period change, the modification of the polarization direction induces only small effects on the spectral response: small spectral shifts and intensity variations.

To end this study, we present the influence of the permittivity of the in-apertures medium $\epsilon_{0}$ on the spectral response of a silver grating. Fig. 8 show the spectral responses for four different in-

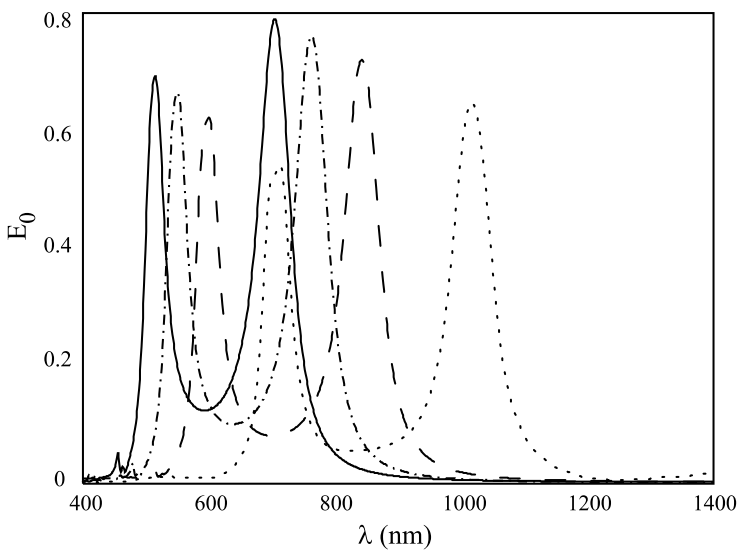

Fig. 8. Influence of the permittivity of the in-apertures medium $\varepsilon_{0}$ on the spectral response of a the silver array of Fig. 2(a). Solid line for $\varepsilon_{0}=1$, dot-dashed line for $\varepsilon_{0}=1.2$, dashed line for $\varepsilon_{0}=1.5$ and dotted line for $\varepsilon_{0}=2.25$. 
apertures media when the other parameters remain the same as in Fig. 2(a).

Once more, the two peaks structure is obtained but large red shifts occur when $\epsilon_{0}$ is increased. These shifts are accompanied by significant decreasing of the intensities. These results are similar to what was obtained by Krishnan et al. (Fig. 2 of [5]) in the case of a circular aperture array made in gold.

\section{Conclusion}

In conclusion, we have numerically studied the spectral responses of a subwavelength annular aperture arrays exhibiting an extraordinary transmission. Several parameters were modified in order to understand the origin of this enhancement. We demonstrate the presence of guided mode through the apertures but we are not able to point out the nature of this mode (is it a TEM one?), neither how it is created. Many others numerical simulations will be necessary to completely understand the phenomenon. Comparisons with others methods, like modal method 9 and references therein], should be useful.

Finally, independently of the interpretation of the enhanced transmission, it is important to underline that the proposed structure could have very interesting applications. The calculation of the near-field above the grating and of the field inside the cavities is too long to be included in this paper. We only mention that the field amplitude is very large and can reach 600 times the incident amplitude. The sensibility of the peaks position with respect to the cavity index, in addition to the existence of a very high electric field amplitude, can lead to interesting phenomena if non-linear material is placed in the apertures.

\section{References}

[1] T.W. Ebbesen, H.J. Lezec, H.F. Ghaemi, T. Thio, P.A. Wolff, Nature 391 (1998) 667.

[2] U. Schröter, D. Heitmann, Phys. Rev. B 58 (1998) 15419.

[3] H.F. Ghaemi, T. Thio, D.E. Grupp, T.W. Ebbessen, H.J. Lezec, Phys. Rev. B 58 (1998) 6779.

[4] J.A. Porto, F.T. Garcia-Vidal, J.B. Pendry, Phys. Rev. Lett. 83 (1999) 2845.

[5] A. Krishnan, T. Thio, T.J. Kim, H.J. Lezec, T.W. Ebbesen, P.A. Wolff, J. Pendry, L. Martin-Moreno, F.J. GarciaVidal, Opt. Commun. 200 (2001) 1.

[6] T. Lopez-Rioz, D. Mendoza, F.J. Garcia-Vidal, J. Sanchez-Dehesa, B. Pannetier, Phys. Rev. Lett. 81 (1998) 665.

[7] Ph. Lalanne, J.P. Hugonin, S. Astilean, M. Palamaru, K.D. Möller, J. Opt. A: Pure Appl. Opt. 2 (2000) 48.

[8] S. Astilean, Ph. Lalanne, M. Palamaru, Opt. Commun. 175 (2000) 265.

[9] E. Popov, M. Nevière, S. Enoch, R. Reinisch, Phys. Rev. B 62 (2000) 16100.

[10] T.-K. Wu, S.-W. Lee, IEEE Trans. Antennas Propag. 42 (1994) 1484

[11] C. Winnewisser, F. Lewen, J. Weinzierl, H. Helm, Appl. Opt. 38 (18) (1999) 3961.

[12] L.D. Landau, E.M. Lifshitz, Electrodynamics of Continuous Media, Pergamon Press, Oxford, UK, 1960.

[13] Allen Taflove, Susan Hagness, Computational Electrodynamics: The Finite-Difference Time-Domain Method, second ed., Artech House, Boston, MA, 2000.

[14] M.N.O. Sadiku, Numerical Techniques in Elelectrodynamics, second ed., CRC Press, Boca Raton, 2000.

[15] G. Mur, IEEE Trans. Electromagn. Compat. 23 (1981) 377.

[16] G. Parent, D. Van Labeke, F.I. Baida, J. Microsc. 202 (2) (2001) 296.

[17] M. Li, J. Wang, L. Zhuang, S.Y. Chou, Appl. Phys. Lett. 76 (6) (2000) 673.

[18] M. Spajer, G. Parent, C. Bainier, D. Charraut, J. Microsc. 202 (2001) 45. 